\title{
Criterios orientadores de la incorporación de condiciones generales en los contratos financieros y la tutela del consumidor peruano*
}

\author{
Olga Alejandra Alcántara Francia ${ }^{a}$
}

\begin{abstract}
Resumen: Este artículo busca identificar los criterios aplicados por la autoridad administrativa que determinan la incorporación de condiciones generales en los contratos financieros celebrados con consumidores. La aprobación administrativa previa del condicionado general se orienta a minimizar los riesgos de inclusión de cláusulas abusivas y a restablecer el equilibrio contractual. Se utiliza una metodología hermenéutico-sintética para la interpretación y el análisis de las resoluciones administrativas emitidas por la autoridad competente. El desarrollo de la investigación arroja que la multiplicidad de criterios jurisprudenciales aplicados a diferentes contratos sujetos a supervisión denota la necesidad de sistematizarlos a fin de garantizar la predictibilidad tanto para el administrado como para el órgano administrativo.
\end{abstract}

Palabras clave: aprobación administrativa; condiciones generales; contratos bancarios; control de incorporación

Fecha de recepción: 14 de septiembre de 2019 Fecha de evaluación: 23 de octubre de 2019 Fecha de aprobación: 28 de octubre de 2019

Cómo citar: Alcantara Francia, O. A. (2020). Criterios orientadores de la incorporación de condiciones generales en los contratos financieros y la tutela del consumidor peruano. Prolegómenos, 23(45). https://doi.org/10.18359/prole.4285

* Este artículo es producto de la investigación del proyecto “La incorporación de condiciones generales en los contratos con consumidores en el derecho peruano y español", financiado por la Universidad de Lima (Perú).

Artículo de revisión.

a Profesora titular de Derecho Civil de la Facultad de Derecho de la Universidad de Lima (Perú). Investigadora adscrita al Instituto de Investigación Científica (IDIC) de la Universidad de Lima (Perú), Magíster en Droit à finalité specialisée en Droit Européen, Université Catholique de Louvain-la-Neuve (Bélgica); DEA en Derecho Civil por la Universidad Carlos III de Madrid, España; Magíster en Derecho Empresarial por la Universidad de Lima (Perú). Correo electrónico: oalcanta@ulima.edu.pe 


\title{
Guiding Criteria for Incorporating General Terms and Conditions in Financial Service Agreements and Protecting the Peruvian Consumer
}

\begin{abstract}
This article seeks to identify the criteria used by the administrative authority to determine the incorporation of general terms and conditions in financial service agreements entered into with consumers. The prior administrative approval of the general terms and conditions aims at minimizing the risks of including abusive clauses and restoring the contractual balance. A hermeneutic-synthetic method is used for interpreting and analyzing administrative resolutions passed by the competent authority. The results show that multiple case-law criteria applied to agreements subject to supervision denote the need for systematizing them to guarantee predictability for both the administered party and the administrative body.
\end{abstract}

Keywords: Administrative approval; general terms and conditions; banking service agreements; incorporation control.

\section{Critérios orientadores da incorporação de condições gerais nos contratos financeiros e na tutela do consumidor peruano}

\begin{abstract}
Resumo: Este artigo pretende identificar os critérios aplicados pela autoridade administrativa que determinam a incorporação de condições gerais nos contratos financeiros firmados com consumidores. A aprovação administrativa prévia das condições gerais é orientada a amenizar os riscos de inclusão de cláusulas abusivas e a reestabelecer o equilíbrio contratual. É utilizada uma metodologia hermenêutico-sintética para interpretar e analisar as resoluções administrativas emitidas pela autoridade competente. O desenvolvimento da pesquisa demonstra que a multiplicidade de critérios jurisprudenciais aplicados a diferentes contratos sujeitos à supervisão denota a necessidade de sistematizá-los, a fim de garantir a previsibilidade tanto para o administrado quanto para o órgão administrativo.
\end{abstract}

Palavras-chave: aprovação administrativa; condições gerais; contratos bancários; controle de incorporação. 


\section{Introducción}

Las condiciones generales de contratación (CGC) son expresiones de la estandarización contractual $y$, por ende, resultado de la masificación a nivel productivo. Al igual que ocurre con los contratos de adhesión, se orientan a la reducción de costos de transacción (Bullard, 1996), de modo que delimitan el contenido de una serie indefinida de contratos. La negociación entre contratantes se elimina en aras de la rapidez o fluidez del intercambio de bienes y servicios. Como bien señala Pagador López (2012),

desde un punto de vista económico su utilización responde a necesidades de racionalización de la actividad contractual de empresarios y profesionales, a los que permite ahorrar tiempo y dinero el que les costaría la negociación singular de cada contrato- y optimizar su organización interna y la utilización de sus recursos (pp. 99-140).

No obstante, las ventajas que brindan estas modalidades para contratar también pueden convertirse en fuente de una serie de situaciones que conlleven la inobservancia de requisitos mínimos que garantizan el respeto de los derechos del consumidor (adherente), lo que provoca el desequilibrio contractual entre las partes o la eventual existencia de "cláusulas abusivas" (Rodríguez García, 2015).

El derecho no puede ser ajeno a esta realidad. De ahí la justificación de diferentes mecanismos de control destinados a garantizar el equilibrio contractual entre consumidor (adherente) y proveedor (empresario, profesional). Uno de estos mecanismos es el control de incorporación, el cual supone, por parte del predisponente, el respeto de una serie de requisitos formales para que las condiciones generales puedan integrar un contrato (Cañizares, 2015; Diez-Picazo, 2007). A este se suman el control de contenido, de transparencia y el control abstracto, cuya operatividad se despliega durante la fase de ejecución del contrato y no de formación.

El objetivo del control de incorporación es garantizar al adherente el acceso a la información relativa al contenido del contrato, de modo que pueda disponer de ciertos datos relevantes que le permitan decidirse a contratar.

En este contexto, la legislación peruana ha optado por un procedimiento de aprobación administrativa ex ante, de condiciones generales, a través de la participación de organismos reguladores de la inversión privada en ciertos sectores "sensibles", como, por ejemplo, el de las telecomunicaciones, el de la banca y el de los seguros. En el ámbito de aquellos contratos no sujetos a supervisión estatal, tales como los contratos de playa de estacionamiento, de transporte privado de pasajeros y, en general, todo otro contrato celebrado o no con consumidores, prima la autonomía privada. La intervención estatal, en algunos casos, se manifiesta a través de la imposición de restricciones o limitaciones respecto al contenido contractual mediante leyes especiales, pero no con la exigencia de un procedimiento de aprobación administrativa. El régimen normativo está constituido por el Código Civil (régimen general) y por el Código de Protección y Defensa del Consumidor (CPDC).

De esta manera, este artículo busca identificar los criterios aplicados por la autoridad administrativa que determinan la incorporación de condiciones generales en los contratos bancarios o financieros celebrados con consumidores. La aprobación administrativa previa es un procedimiento complejo que se orienta, por medio de una evaluación y calificación previa, a minimizar los riesgos de inclusión de cláusulas abusivas, así como a restablecer el equilibrio contractual. Para tanto, se recurrió a las resoluciones administrativas de la autoridad competente: la Superintendencia de Banca, Seguros y Administradoras de Fondos de Pensiones (sBs). A través de estas se ha determinado que la multiplicidad de criterios jurisprudenciales aplicados a diferentes contratos sujetos a supervisión denota la necesidad de sistematizarlos a fin de garantizar la predictibilidad tanto para el administrado como para el órgano administrativo. La utilización del método descriptivo y del método hermenéutico-sintético ha permitido interpretar y analizar las resoluciones administrativas emitidas por la autoridad competente. 


\section{A. La aprobación administrativa previa de condiciones generales y la intervención del Estado en la contratación financiera}

En una sociedad en la que las reglas del mercado determinan quién entra o quién sale de este, quién vende más o quién vende menos, resulta más conveniente para el empresario distribuir sus productos o servicios mediante contratos uniformizados u homogéneos (Slawson, 1971) en los que se pueda minimizar la mayor cantidad de riesgos, lo que incluye el de las reclamaciones judiciales o de otra índole. Para algunos, la decisión de intervenir en la contratación estandarizada al controlar que los empresarios o proveedores incluyan determinadas cláusulas en los formularios contractuales puede ser nociva, pues "no hace otra cosa que imponer [...] diversos riesgos no deseados" (Escobar Rozas, 2011, p. 267). Sin embargo, el legislador peruano ha considerado necesaria la intervención en los contratos de consumo a través de procedimientos administrativos de aprobación de condiciones generales de contratación (CGC) que determinan su incorporación en contratos masivos, tales como los bancarios o de telecomunicaciones, así como al ejercer un control de contenido (con el establecimiento de una lista gris y una lista negra en el Código de Protección y Defensa del Consumidor [CPDC] para la determinación de cláusulas abusivas). Ambos tipos de control se ejercen sobre las cláusulas principales y accesorias del contrato.

La doctrina ha considerado que la efectividad de la obligación de informar a los consumidores no garantiza que estos vayan a leer o que deseen conocer la información sobre el contrato que van a firmar y, de hecho, muchas veces así ocurre, pues el consumidor puede encontrarse con barreras que le impiden obtener información relevante o simplemente se muestran indiferentes frente a las CGC por su falta de claridad o su elevado tecnicismo (Ben-Shahar, 2009; Marotta-Wrugler, 2005).

Este contexto nos lleva a pensar que la obligación de informar es insuficiente, tanto como el mero establecimiento de requisitos vinculados a la redacción o de carácter formal, ya que no consiguen que la lectura del contenido contractual se convierta en una actividad relevante en la toma de decisiones del consumidor (Korobkin, 2003; Thaler, 2003). En esta medida no resulta ilógica una solución que admite la intervención del Estado a través de órganos administrativos que ahorran al consumidor los costos de búsqueda de información - de transacción - y de oportunidad, pero que, además, le brinda seguridad jurídica pues ejercerá un control formal y de legalidad sobre las CGC de determinados contratos masivos que son altamente técnicos y complejos, como, por ejemplo, los relacionados con las telecomunicaciones y los de naturaleza bancaria y financiera. Se ha establecido así en el derecho peruano un procedimiento administrativo de aprobación CGC a través de la participación de autoridades administrativas con competencia específicas sobre la materia.

Estos órganos administrativos han sido creados por leyes especiales bajo la denominación de "organismos supervisores de la inversión privada" (Ley N.o 27332, D.Leg. 702). Así, para los contratos propios del sector bancario, financiero y de seguros, la autoridad encargada es la Superintendencia de Banca y Seguros y Administradoras Privadas de Pensiones"; para el sector de telecomunicaciones el órgano administrativo encargado es el Osiptel $^{2}$ (organismo supervisor de inversiones en telecomunicaciones), en lo que concierne a contratos celebrados de forma directa con consumidores

1 La Superintendecia de Banca y Seguros y Administradoras Privadas de Fondos de Pensiones (sbs) supervisa las actividades de las empresas reguladas por la Ley N. ${ }^{\circ}$ 26702. Por Resolución sbs N. ${ }^{\circ} 8181-$ 2012, del 25 de octubre de 2012, se aprobó el Reglamento de Transparencia de Información y Contratación con usuarios del Sistema Financiero, y por Resolución sbs 3274-2017 se aprobó el Reglamento de Gestión de Conducta del Mercado y Sistema Financiero, así como modifican el Manual de Contabilidad para las empresas del sistema financiero y el Reglamento de Tarjetas de Crédito y Débito.

2 El Osiptel cuenta con un texto único ordenado (tuo) de las Condiciones de Uso de los Servicios Públicos de Telecomunicaciones aprobado por Resolución

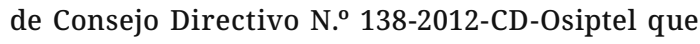
entró en vigencia desde el 1 de octubre de 2012. 
y usuarios. Para efectos de este trabajo, el objeto de estudio se ha centrado de manera exclusiva en la aprobación de las CGC en los contratos bancarios, por ser estos los que presentan mayor incidencia en reclamos ${ }^{3}$.

La mencionada aprobación administrativa de los contratos relativos a ofertas de productos o servicios se realiza antes de lanzarlos al mercado. El objetivo es verificar "la existencia de equilibrio entre los derechos y obligaciones del proveedor predisponente y de los consumidores" (Torres Vásquez, 2016, p. 556). La incorporación automática de las CGC al contrato se producirá siempre y cuando el ente regulador haya dado su conformidad a través de un procedimiento administrativo. Por una parte, se verifica que las CGC respeten los requisitos formales establecidos en el CPDC, y, por otra, se revisa si el contenido de las CGC respeta los derechos mínimos de los consumidores o usuarios previstos en el CPDC y en la normativa sectorial bancaria (control de legalidad). Se someten a este tipo de control las CGC que regulen tanto los elementos esenciales como los elementos accesorios del contrato (O’Callaghan Muñoz, 2004), no obstante, no se interviene en la fijación de precios o tarifas de los servicios.

La accesibilidad y la publicidad de las CGC son necesarias para que se produzca la incorporación automática indicada (art. 6, TUO Osiptel, art. 13, Reglamento de Transparencia SBS), pues de probarse lo contrario esta no ocurrirá. Se puede afirmar, entonces, que el procedimiento de aprobación administrativa garantiza la libertad de decisión negocial del consumidor debidamente informado.

Los reglamentos de transparencia o aquellos que establecen "condiciones de uso" constituyen una pauta básica para los proveedores en la redacción de sus condicionados generales. De ahí que a través de resoluciones administrativas, emitidas por los organismos reguladores, se excluyan o

3 Un total de 45 de cada 100 reclamos presentados por consumidores son relativos a servicios bancarios, de acuerdo con la estadística elaborada por el Servicio de Atención al Ciudadano para el periodo marzo 2016-abril 2017. rechacen aquellas CGC que no garanticen una "información mínima" al consumidor.

En lo que concierne a las entidades sometidas al procedimiento administrativo de aprobación de CGC se encuentran las empresas pertenecientes al sistema financiero, al sistema de seguros, al sistema privado de pensiones y a las cooperativas de ahorro y crédito. El sistema financiero es el que concentra el mayor número de empresas, tales como los bancos, las cajas rurales de ahorro y crédito, las cajas municipales, las edypimes, las empresas afianzadoras de garantías, las empresas de servicios fiduciarios y las empresas de arrendamiento financiero. En este sentido, los requerimientos de aprobación administrativa de CGC son más numerosos, en proporción, a los de las empresas que forman parte de los otros sistemas supervisados.

De otra parte, cabe agregar que los consumidores o usuarios pueden denunciar una o más cláusulas por considerarlas abusivas y someterlas a un control de contenido ${ }^{4}$ ante las instancias administrativas especializadas en protección al consumidor: la Comisión de Protección al Consumidor (CPC) y la Sala Especializada en Protección al Consumidor (Sala) del Instituto Nacional de Defensa de la Competencia y de la Propiedad Intelectual (Indecopi). Esto implica la coexistencia de un doble control estatal: antes de la conclusión del contrato y durante su ejecución. Aunque cabe indicar que, en la práctica, el control de contenido practicado por el Indecopi sobre contratos que incluyen CGC recae, más bien, en aquellas cláusulas predispuestas no sometidas ni a negociación ni incluidas en el condicionado general.

4 "Artículo 43. Reglamento de Transparencia. sbs. La aprobación de cláusulas generales de contratación, así como la determinación de cláusulas abusivas, no impide a los usuarios recurrir ante las instancias administrativas y judiciales que correspondan a fin de salvaguardar sus derechos". 


\section{B. Régimen normativo general y sectorial aplicable al procedimiento administrativo de aprobación de CGC}

\section{Normas contenidas en el Código de Protección y Defensa del Consumidor (CPDC)}

El Indecopi es la institución estatal que tiene competencia primaria para aplicar las normas contenidas en el CPDC por medio de procedimientos sancionadores seguidos en contra de los proveedores (empresas, profesionales) y denunciados por los consumidores. De manera general, esta institución vela porque el derecho a la información, así como todo el catálogo de derechos mínimos de los consumidores, estén garantizados en todo tipo de contrato. Para esto, los artículos $47^{5}$ y $48^{6}$ establecen una serie de obligaciones destinadas a asegurar el suministro y la difusión de información sobre el contenido contractual. De igual modo, las exigencias de tipo formal que busca garantizarle al consumidor el estar informado antes de contratar la adquisición de algún producto o servicio. La expresión de la voluntad inequívoca del consumidor,

5 “Artículo 47. Protección mínima del contrato de consumo. En los contratos de consumo se observa lo siguiente:

1. En los contratos cuyas condiciones consten por escrito o en algún otro tipo de soporte, debe constar en forma inequívoca la voluntad de contratar del consumidor. Es responsabilidad de los proveedores establecer en los contratos las restricciones o condiciones especiales del producto o servicio puesto a disposición del consumidor.

2. No pueden incluirse cláusulas o ejercerse prácticas que impongan obstáculos onerosos o desproporcionados para el ejercicio de los derechos reconocidos al consumidor en los contratos.

3. Los consumidores tienen derecho a emplear los mismos mecanismos de forma, lugar y medios utilizados para la celebración de los contratos para desvincularse de estos, como por ejemplo la vía telefónica, cualquier medio electrónico u otro análogo. Esta facultad comprende la contratación en cualquier tipo de soporte y como elemento de validez del contrato, así como la prohibición de incluir cláusulas que conlleven la desproporción entre las obligaciones y los derechos asumidos por las partes, así como el tamaño de letra en la cual deben expresarse las condiciones, son exigencias válidas para todo tipo de contrato bien sea este sujeto a aprobación administrativa o no. El artículo 48 está referido a un conjunto de requisitos de incorporación aplicables a las condiciones generales que integrarán todo tipo de contrato sujeto o no a supervisión. Nadie duda que las cláusulas deben redactarse con claridad y sencillez, es decir, ser comprensibles y legibles de modo tal que el consumidor pueda entenderlas antes de la celebración del contrato y, en principio, no le quede dudas con respecto a lo que firma.

Asimismo, el CPDC, en sus artículos 53 a 55, reconoce la competencia de los organismos reguladores para aprobar administrativamente los condicionados generales relativos a servicios públicos, tales como la SBs (art. 54.2). El procedimiento de aprobación administrativa, realizada por la SBS, se rige por las normas del sector y las disposiciones del CPDC en lo que a protección de consumidores concierna.

de prestaciones adicionales o complementarias.

4. En el caso de formularios contractuales, los caracteres de estos deben ser adecuadamente legibles para los consumidores, no debiendo ser inferiores a tres (3) milímetros. La redacción y términos utilizados deben facilitar su comprensión por los consumidores.

Los proveedores deben entregar a los usuarios copia de los contratos y demás documentación relacionada con dichos actos jurídicos cuando estos hayan sido celebrados por escrito, incluidas las condiciones generales de la contratación. Los proveedores son responsables de dejar constancia de la entrega de los documentos al consumidor. En el caso de contratación electrónica, el proveedor es responsable de acreditar que la información fue puesta oportunamente a disposición del consumidor. No son exigibles las cláusulas, condiciones, estipulaciones y prácticas que infrinjan el presente artículo”. 


\section{Legislación especial del sector financiero}

ley que regula la intervención de la sBs en los contratos masivos, financieros, de seguros y de administradoras de fondos de pensiones es la Ley 28578 (ley complementaria a la ley de protección al consumidor en materia de servicios financieros). Esta norma señala de manera expresa que la aprobación administrativa tiene por "finalidad establecer el equilibrio contractual necesario entre las empresas y los usuarios, garantizar la operatividad del servicio financiero y el respeto de los usuarios". Esto no implica, de ninguna manera, la invariabilidad de las condiciones contractuales. Para que sean oponibles al usuario las modificaciones contractuales deberán comunicarse estas de manera fehaciente. Por lo general, todas las disposiciones de esta ley se orientan a garantizar el derecho a la información de los usuarios, como, por ejemplo, de las comisiones, las tasas de interés, los gastos y sus respectivas liquidaciones.

Antes de la entrada en vigencia del CPDC, la SBS aprobó el Reglamento de Transparencia de Información y las disposiciones aplicables a la contratación con usuarios del sistema (Resolución SBS N. ${ }^{1765-2005) . ~ E s t e ~ r e g l a m e n t o ~ a r m o n i z a b a ~ c o n ~}$ las disposiciones de la Ley de Protección al Consumidor, vigente en ese momento. En dicho documento se establecían los criterios aplicables para la determinación de las tasas de interés, las comisiones y los gastos, así como su difusión en soporte físico o a través de los portales web. Se incluyó, además, un capítulo dedicado a la información que se debe proporcionar al usuario para efectos

6 “Artículo 48. Requisitos de las cláusulas contenidas en un contrato de consumo por adhesión. En los contratos de consumo celebrados por adhesión o con cláusulas generales de contratación, debe cumplirse con los siguientes requisitos:

1. Concreción, claridad y sencillez en la redacción, con posibilidad de comprensión directa, sin reenvíos a textos o documentos que no se faciliten previa o simultáneamente a la conclusión del contrato, y a los que, en todo caso, debe hacerse referencia expresa en el documento contractual. de la contratación y la prestación de operaciones activas, pasivas y de servicios. Se impuso a la empresa financiera la obligación de absolver todas las dudas o cuestionamientos que pudieran tener los usuarios (art. 12), así como de entregarle al consumidor una hoja resumen (anexo que contiene toda la información con respecto a los intereses, las comisiones y los gastos debidamente firmada por la persona autorizada de la empresa). El artículo 15 explicita toda la información que esta hoja resumen debe contener.

Más adelante, cuando el CPDC se encontraba vigente (pues entró en vigencia en el 2010 con la Ley 29571), la Resolución sBs N. o 8181-2012 aprueba el nuevo Reglamento de Transparencia de Información y Contratación con los usuarios del sistema financiero, lo cual armoniza su contenido con el CPDC. El objetivo de este reglamento, al igual que su predecesor, es alcanzar la transparencia en la difusión, la aplicación y la modificación de las condiciones contractuales, las tasas de interés compensatorio, moratorio o las penalidades aplicables en caso de incumplimiento, las comisiones y los gastos asociados a las operaciones activas y pasivas, así como a los servicios que brinden las empresas del sistema financiero. Con la transparencia se mejora el acceso a la información de los usuarios y así se les garantiza una toma de decisión de consumo informada. Del mismo modo que en el anterior reglamento, se precisan los criterios aplicables para la determinación de las tasas de interés, las comisiones y los gastos; asimismo, la obligación de la empresa financiera de establecer mecanismos de difusión de la información a través de listados informativos, folletos, cajeros automáticos, páginas

2. Accesibilidad y legibilidad, de forma que permita al consumidor y usuario el conocimiento previo del contenido del contrato antes de su suscripción.

3. Buena fe y equilibrio necesario en los derechos y obligaciones de las partes, lo que en todo caso excluye la utilización de cláusulas abusivas.

Lo dispuesto en el presente artículo resulta de aplicación a los contratos celebrados en base a cláusulas generales de contratación, se encuentren o no sometidas a aprobación administrativa”. 
web, etc. Se ratifica, también, la obligación de la empresa financiera de entregar la información contractual de manera previa a la celebración o firma del contrato.

Más recientemente, la Resolución sBs N. ${ }^{\circ} 3274-$ 2017, por la cual se aprueba el Reglamento de Conducta de Mercado del Sistema Financiero, refuerza el objetivo de lograr la transparencia de información en la medida en que es un mecanismo de mejora del acceso a la información de los usuarios. Señala el reglamento que "las empresas deben ser diligentes en la información que bridan respecto de los productos y servicios ofrecidos, con el fin de que los usuarios comprendan sus características, beneficios, riesgos y condiciones aplicables, y puedan tomar decisiones de consumo informadas". Este reglamento abarca la gestión de reclamos y la necesidad de que las respuestas sean comprensibles y sencillas, pero, además, se ocupa de la gestión de la conducta de mercado, lo que hace responsables a las empresas en el desarrollo de políticas de cultura organizacional (artículo 4).

\section{Facultades de la SBS en el marco del procedimiento administrativo de aprobación de las CGC}

De la revisión de la jurisprudencia administrativa que abarca desde el 2008 hasta el 2019 se ha observado que la sBs está facultada para pronuciarse sobre cualquier producto o servicio dirigido a los usuarios (o consumidores) protegidos por el CDPC (Resolución SBS N.o 2622-2015; Resolución SBS N.o 2184-2019; Resolución sBs N. $3626-2019)$. Las empresas financieras deben someter a aprobación administrativa previa (Resolución SBS N.o 3482-2014) las CGC relacionadas con las siguientes operaciones y servicios:

- Operaciones activas. Crédito hipotecario y crédito de consumo (Resolución sBs N. . 3233-2009; Resolución sBs N. ${ }^{\circ} 7017-2009$; Resolución sBs N. ${ }^{\circ}$ 1101-2009; Resolución sBs N. ${ }^{\circ}$ 1449-2009; Resolución SBS N.o 7835-2009; Resolución SBS N. ${ }^{\circ} 10519$ 2009; Resolución sBs N.o 9871-2009).

- Operaciones pasivas. Depósito de ahorro, depósito a plazos, depósito por compensación de tiempo de servicios, depósito en cuenta corriente (Resolución SBS N. ${ }^{\circ}$ 2185-2009; Resolución SBS N. ${ }^{\circ}$
10367-2009; Resolución sBs N. o 8948-2009; Resolución sBs N. ${ }^{9}$ 9756-2008).

- Servicios. Contrato de cajas de seguridad y contrato de custodia (Resolución sBs N. ${ }^{\circ}$ 4552-2009; Resolución sBs N.o 4708-2009).

Cabe agregar que este listado es enunciativo, pues la SBS está facultada para someter al procedimiento de aprobación administrativa previa a las CGC de cualquier otro producto o servicio que se pueda pactar con los usuarios sujetos a la protección del CDPC. Es así que las CGC de los contratos relacionados con las cuentas de dinero electrónico Bim también están sujetos a supervisión y aprobación administrativa (Resolución sBS N. ${ }^{\circ}$ 22022019; Resolución SBS N. . 2189-2019; Resolución SBS N. 2201-2019; Resolución sBs N.o 933-2019; Resolución SBS N. ${ }^{\circ} 2000-2018$ ).

Ahora bien, desde hace algunos años la sBs ha venido centrando su atención en las condiciones generales de contratación que regulan los siguientes aspectos:

- resolución del contrato por causal distinta al cumplimiento;

- conclusión del contrato de manera anticipada;

- limitación o exoneración de responsabilidad por parte de la empresa;

- limitación o exclusión de los derechos de los usuarios, y

- centralización de las cuentas del cliente, salvo que se trate del derecho de compensación.

Quedan excluidas de cualquier pronunciamiento de la sBs las condiciones generales relacionadas con normas que se encuentren en el ámbito de competencia de otros organismos como, por ejemplo, la Autoridad Nacional de Datos Personales o el Indecopi (Resolución sBs N. o 4435-2016).

Asimismo, la sBS está facultada por ley (art. 46, Reglamento de Transparencia de Información y las disposiciones aplicables a la contratación con usuarios en el sistema financiero) para, en el marco del procedimiento administrativo de aprobación de CGC, identificar las cláusulas que, eventualmente y dadas sus características, pudieran considerarse abusivas (artículo 54.5, CDPC) (Resolución SBS N. ${ }^{\circ}$ 8253-2014; Resolución sBS N. ${ }^{\circ}$ 1942-2015; Resolución sBS N.o 5942-2016; Resolución sBS N. ${ }^{\circ}$ 
7333-2015; Resolución sBs N. ${ }^{\circ}$ 7126-2015; Resolución sBS N. ${ }^{\circ}$ 5254-2013; Resolución sBS N. ${ }^{0}$ 57922014; Resolución sBs N. . 5791-2014; Resolución SBS N. 5228-2014; Resolución sBs N. ${ }^{\circ}$ 6250-2014; Resolución sBs N.o 4806-2015; Resolución sBs N.o 70692015; Resolución sBs N. ${ }^{\circ}$ 3522-2016; Resolución SBS N. ${ }^{\circ}$ 6860-2013; Resolución sBs N.o 1066-2015).

Finalmente, cabe agregar que la sBs está facultada para requerir en el futuro una nueva revisión de las CGC aprobadas (numeral 8, artículo 349 de la Ley General del Sistema Financiero y del Sistema de Seguros y Orgánica de la SBS) (Resolución sBS N. ${ }^{\circ}$ 1684-2018; Resolución sBs N. ${ }^{\circ}$ 357-2018; Resolución sBs N. ${ }^{\circ} 4050-2014$; Resolución sBs N. ${ }^{\circ} 942-$ 2015; Resolución sBS N. 3 3535-2014; Resolución SBS N.o 5617-2013; Resolución sBs N. ${ }^{\circ}$ 10580-2008; Resolución sBs N.o 3481-2014; Resolución sBs N. ${ }^{\circ}$ 1820-2017; Resolución sBs N. ${ }^{\circ}$ 2122-2018; Resolución sBS N.o 8149-2014; Resolución sBS N. ${ }^{0}$ 55752016; Resolución sBs N. ${ }^{\circ}$ 7778-2014; Resolución SBS N. $3819-2014)$.

\section{Criterios orientadores de la aprobación administrativa y la protección al consumidor}

\section{Criterios relativos a la tutela del derecho a la información}

Al momento de evaluar las CGC de los diferentes contratos relativos a productos o servicios presentados por las entidades del sistema financiero, la sBs busca garantizar el suministro de información sobre los costos, las comisiones y los demás gastos que deba asumir el consumidor relacionados con la operación financiera por realizar o realizada. En este sentido, es importante que la hoja de resumen, la cual forma parte integrante del contrato, contenga los costos trasladados al usuario de forma previa a la conclusión del contrato, sin perjuicio de la información permanente puesta al alcance del consumidor bajo diferentes modalidades (Resolución sBs N.o 13055-2009).

Ahora bien, es de vital importancia que las entidades financieras identifiquen (Resolución sBS N. ${ }^{13006-2009 ;}$ Resolución sBs N.o 13007-2009) e informen al usuario acerca de los medios de comunicación a través de los cuales se le comunicará acerca de las variaciones contractuales decididas de forma unilateral, más aún cuando conllevan la variación de tarifas y/o costos asociados a las operaciones realizadas (Resolución sBs N. ${ }^{\circ} 13055$ 2009). Todo esto sin perjuicio de la información de carácter permanente que la entidad financiera ponga a disposición de los usuarios, en general, a través de los distintos medios de comunicación implementados para dicho efecto. En esta misma línea no es admisible la existencia de cláusulas referidas al uso exclusivo de mecanismos de información ajenos al contrato a efectos de informar condiciones aplicables a él, sin hacer referencia a la necesidad de que tales términos y/o condiciones consten en el texto mismo del contrato, incluidos sus anexos (Resolución sBs N. ${ }^{9}$ 9864- 2009).

De manera general, la sBs no admite las cláusulas que obliguen al usuario a aceptar términos o condiciones no conocidas o no informadas al momento de formular su aceptación (Resolución sBS N. ${ }^{\circ}$ 13006-2009; Resolución SBS N. ${ }^{\circ}$ 13007-2009; Resolución SBS N. ${ }^{\circ}$ 130011-2009; Resolución SBS N. ${ }^{\circ}$ 13008-2009; Resolución sBS N. ${ }^{\circ}$ 13613-2009; Resolución SBS N. ${ }^{\circ}$ 13614-2009; Resolución SBS N. ${ }^{\circ}$ 2416-2009).

\section{Suministro de información previa al ejercicio del derecho de compensación a favor de la empresa financiera}

El derecho de compensación reconocido a las sentidades del sistema financiero las faculta a cobrarse las deudas impagas afectando directamente las cuentas de ahorros que los usuarios mantengan en el mismo establecimiento en el que solicitaron el préstamo o crédito. Este derecho está reconocido por el artículo 132 de la Ley 26702 (Ley General del Sistema Financiero, del Sistema de Seguros y Orgánica de la Superintendencia de Banca y Seguros). En este contexto, los condicionados generales deben incluir las condiciones o circunstancias en las que la entidad bancaria ejercerá el derecho de compensación. No es factible incorporar cláusulas que permitan a la entidad finaciera reservarse el ejercicio de este derecho sin informarle al usuario (Resolución 
SBS N. 13006-2009; Resolución SBS N.o 13007-2009; Resolución SBS N. ${ }^{0} 13613-2009$; Resolución SBS N. ${ }^{\circ}$ 13614-2009; Resolución sBs N. 2416-2009).

\section{Obligación de informar al usuario con respecto a la oportunidad del ejercicio del extorno}

La sBs ha señalado, en varias resoluciones, que la cláusula de exoneración de responsabilidad del banco como consecuencia del extorno de operaciones es factible siempre que se haya pactado con antelación a su ocurrencia y haya sido informada al usuario; no es aplicable en los casos en los que la actuación negligente de la empresa estuviera comprometida (Resolución sBs N. ${ }^{\circ}$ 2416-2009; Resolución SBS N. ${ }^{\circ}$ 2655-2009).

\section{Obligación de informar al usuario} sobre las tasas de interés, las comisiones, los gastos y las modificaciones contractuales

Todo consumidor o usuario que celebra un contrato de mutuo con garantía hipotecaria o, en general, cualquier contrato de crédito afecto a intereses, comisiones y gastos debe tener la oportunidad de conocer a cuánto ascienden estos, incluso antes de contratar. En tal sentido, si estos son variables o sujetos a modificación por parte de la empresa financiera deben de ser, necesariamente, informados a los usuarios. No resulta admisible la incorporación de cláusulas que facultan a la empresa financiera a modificar, de manera discrecional, (Resolución SBS N. ${ }^{\circ}$ 13614-2009) los términos contractuales sin garantizar el uso de medios de comunicación adecuados (Resolución sBs N.o 9864-2009; Resolución SBs N. ${ }^{\circ}$ 13006-2009). Estos medios de comunicación deben incluir canales directos; solo en defecto de estos se podrán utilizar los medios masivos de comunicación (Resolución sBs N. ${ }^{0}$ 12340-2009; Resolución SBs N. ${ }^{\circ}$ 130011-2009).

En este contexto, no es admisible la cláusula que permite la resolución intempestiva del contrato sin expresión de causa y sin plazo de preaviso. Se debe garantizar en este tipo de cláusulas el conocimiento del cliente respecto a la causal aplicada en su caso concreto (Resolución SBS N. ${ }^{\circ}$ 12340-2009; Resolución sBs N. ${ }^{0} 13006-2009$; Resolución sBs N.o 13007-2009; Resolución sBs N. . 130011-2009; Resolución SBS N. ${ }^{\circ}$ 13613-2009; Resolución SBS N. ${ }^{\circ}$ 2416-2009).

\section{Obligación de informar al usuario en caso de bloqueo de cuentas o retención por medida cautelar}

La SBs ha señalado que las empresas financieras deben garantizar, en el marco de sus CGC, el derecho del usuario a estar informado acerca de las medidas de bloqueo o de retención en cuenta derivadas de la aplicación de medidas preventivas o cautelares (Resolución sBs N. ${ }^{\circ}$ 2655-2009).

\section{Suministro de información crediticia del cliente}

Las CGC deben precisar y delimitar el alcance de la facultad - a favor del banco- de proporcionar información crediticia del cliente a agentes de información crediticia o terceros con legítimo interés con respecto a su comportamiento crediticio (Resolución sBs N. 12340-2009; Resolución sBS N. $\left.{ }^{\circ} 1425-2011\right)$.

\section{Obligación de informar sobre la contratación de servicios de cobertura}

La sBs ha denegado la incorporación de CGC que tengan por finalidad exigir la contratación de servicios de cobertura sin garantizar aquella información mínima de carácter esencial que exigen las disposiciones vigentes (Resolución sBs N. ${ }^{\circ}$ 12340-2009).

\section{En torno a la tutela de los derechos mínimos del consumidor}

\section{Reconocer el derecho del consumidor a efectuar "prepagos" sin condicionamientos}

El derecho del consumidor a realizar pagos anticipados o "prepagos" está consagrado en el artículo 1.1.k del CPDC. Este derecho le permite al 
consumidor, en toda operación de crédito, pagar de forma total o parcial su deuda, y tener derecho a la reducción de los intereses compensatorios generados al día del pago, así como a la liquidación de las comisiones y gastos derivados de las cláusulas contractuales pactadas entre las partes sin que pueda serle aplicable ningún tipo de penalidad o cobro adicional o similar.

Las CGC aprobadas administrativamente no pueden contener cláusulas que limiten o impongan condiciones al ejercicio del derecho de prepagar las deudas que tengan a su cargo los consumidores (Resolución sBs N.o 13616-2009; Resoución sBs N.o 13615-2009; Resolución sBs N.o 13007-2009).

\section{Reconocer el derecho de disponer o enajenar los bienes de propiedad del usuario}

La sBs busca garantizar que en los condicionados generales se reconozca a los usuarios su derecho a disponer o gravar los bienes de su propiedad (Resolución sBs N. ${ }^{\circ}$ 13006-2009), así como que tal derecho conste de manera expresa en los formularios y contengan las CGC (Resolución sBS N. ${ }^{\circ} 130088$ 2009; Resolución sBs N.o 131388-2009; Resolución SBs N. ${ }^{\circ}$ 13514-2009; Resolución sBs N.o 14212-2009, Resolución SBs N. ${ }^{\circ}$ 15211-2009; Resolución SBs N. 15564-2009).

\section{En relación con la admisión o el rechazo de poderes presentados por los usuarios}

Al respecto, la sBS no se opone a que las entidades financieras rechacen u observen los testimonios o escrituras públicas, conteniendo poderes, presentados por los usuarios. Lo que no resulta admisible es que no hayan adoptado previamente un procedimiento de revisión estándar que asegure un comportamiento igualitario por parte de las empresas (Resolución sBs N. ${ }^{\circ}$ 13006-2009; Resolución sBS N. ${ }^{\circ}$ 13007-2009; Resolución sBs N.o 136142009; Resolución SBS N. ${ }^{\circ}$ 2416-2009).

\section{Establecimiento de penalidades frente a la imposibildiad de pago en circunstancias no imputables al cliente}

La sBs no admite, sino prohibe, las cláusulas que impongan penalidades por incumplimiento en el pago en situaciones de imposibilidad no atribuibles al usuario, como, por ejemplo, supone penalizar el pago en circunstancias en las que la fecha de vencimiento coincide con los días inhábiles (Resolución sBs N. ${ }^{0}$ 1425-2011).

\section{En cuanto a la eficacia del contrato, derechos de terceros y exoneración de responsabilidad por fuerza mayor o caso fortuito}

La sBs ha establecido en algunas resoluciones sobre aprobación de CGC de contratos de arrendamiento financiero la necesidad de hacer referencia expresa a la condición de "separabilidad" que regula el Código Civil en materia de nulidad del acto jurídico, a efectos de determinar que la nulidad de una cláusula del contrato no afectará a las demás disposiciones de este (Resolución sBs N. o 14118-2009).

En aras de proteger los derechos de terceros, específicamente del fiador, la sBs no admite cláusulas que limiten el derecho del fiador a oponer excepciones que le correspondan al cliente o deudor principal, o que le impidan liberarse de su obligación por imposibilidad de subrogación (Resolución sBS N. ${ }^{\circ}$ 14118-2009; Resolución sBs N.o 130011-2009).

Asimismo, no es admisible la cláusula que impida la renuncia de los fiadores solidarios y prestatarios, en cualquier momento, al envío de publicidad a través de sus correos electrónicos (Resolución SBS N. ${ }^{\circ}$ 13007-2009).

No es posible, tampoco, incluir cláusulas de exoneración de responsabilidad a favor de la empresa financiera que no resulten ser consecuencia directa de eventos catalogados como de caso fortuito o hechos de fuerza mayor (Resolución SBS N.o 9864-2009; Resolución sBs N.o 2655-2009). Ni tampco las cláusulas que coaccionan al usuario a aceptar y reconocer como propia la realización de transacciones fraudulentas a través de canales de servicios bajo cualquier supuesto (Resolución SBS N.o 9864-2009; Resolución SBs N. ${ }^{\circ}$ 2416-2009). 
La SBS no admite las cláusulas que contengan limitaciones de responsabilidad a favor de la entidad financiera frente a eventuales perjuicios derivados de errores operativos en los que no actúen con la diligencia ordinaria requerida o no cumplan con la obligación a su cargo por circunstancias imputables a estas o a terceros de los que se valen para prestar sus servicios, de modo que queda limitada solo a situaciones de caso fortuito o fuerza mayor (Resolución sBs N. ${ }^{\circ}$ 2655-2009; Resolución SBS N. ${ }^{\circ}$ 2416-2009). Es más, como bien se ha señalado, las restricciones contractuales deben estar basadas en razones fundadas y de carácter objetivo (Resolución SBS N. ${ }^{\circ}$ 1425-2011).

\section{Conclusión}

Dado que resulta costoso y desalentador exigirle al proveedor un contrato completamente simétrico (en términos de información), la autoridad administrativa peruana interviene de forma directa al controlar la incorporación de CGC en los contratos bancarios y financieros a través de la imposición de un procedimiento administrativo de aprobación. Este procedimiento responde a una visión tradicionalista del derecho contractual de consumo y a una decisión proteccionista de parte del Estado peruano, característica de las economías en vías de desarrollo, en las cuales no termina de asentarse una visión que relativice la aparente "vulnerabilidad e inferioridad" del consumidor.

No obstante, con el paso de los años la revisión de la jurisprudencia administrativa emanada de la SBS se ha armonizado con las normas tuitivas de los derechos consumidores contenidas, primero, en la legislación sobre la materia, y, luego, a partir del 2010 en el CPDC. Del análisis y la síntesis de las decisiones emanadas de los procedimientos administrativos se desprende una serie de criterios que orientan la aprobación de CGC. El objetivo de estos criterios es garantizar la transparencia y el acceso a la información del consumidor con respecto al contrato por celebrar. Son sumamente útiles para los operadores (llámese bancos, financieras, edypimes, cajas de ahorro, etc.) porque con su aplicación se excluyen aquellas cláusulas atentatorias contra los derechos del consumidor y/o abusivas.
La revisión de la jurisprudencia administrativa de la sBs de los últimos diez años revela la necesidad de sistematizar los criterios orientadores de las decisiones de la autoridad administrativa a fin de garantizar la transparencia, pero, sobre todo, su predictibilidad, lo cual disminuye el riesgo de observaciones o rechazos de las solicitudes de aprobación administrativa presentadas por las empresas financieras. La publicidad y la difusión del documento que contenga estos criterios sistematizados coadyuvará al logro de esta finalidad.

\section{Referencias}

Ben-Shahar, O. (2009), The Myth of the "Opportunity to Read" in Contract Law. The University of Chicago Law Review, 1, 17. Recuperado de: http://home.uchicago. edu/omri/pdf/articles/The_Myth_Of_Opportunity_ To_Read.pdf

Bullard, A. (1996). Estudios de análisis económico del derecho. Lima: Ara Editores.

Cañizares, A. (2015). Control de incorporación y transparencia de las condiciones generales de la contratación. Las cláusulas suelo. Revista de Derecho Civil, Estudios, II(3), 67-105.

Diez-Picazo, L. (2012). Fundamentos del derecho civil patrimonial (6 $6^{\mathrm{a}}$ ed.). Pamplona: Thomson-Civitas.

Escobar Rozas, F. (2011). Entendiendo el mercado: la contratación estandarizada como forma de mitigar los problemas de insatisfacción y de selección adversa. En O. Súmar (ed.), Ensayos sobre protección al consumidor en el Perú (pp. 257-282). Lima: Universidad del Pacífico.

Korobkin, R. (2003). Bounded Rationality, Standard Form Contracts and Unconscionability. The University of Chicago Law Review, 70, 1234. Recuperado de https:// chicagounbound.uchicago.edu/uclrev/vol70/iss4/2/

Marotta-Wrugler, F. (2005). Are "Pay Now, Terms Later" Contracts Worse for Buyers? Evidence for Software Licence Agreements. The Journal of Legal Studies, 38(2), pp. 309-343.

O’Callaghan Muñoz, X. (2004). Código civil, comentado y con jurisprudencia, Madrid: Editorial La Ley.

Pagador López, J. (2012). Los contratos de consumo celebrados mediante condiciones generales y cláusulas predispuestas. En L. P. Miranda Serrano y J. Pagador López (coords.), Derecho (privado) de los consumidores (p. 99-143). Madrid: Marcial Pons.

Rodríguez García, G. (2015). El equilibrista desorientado: la dinámica evolutiva de las cláusulas contractuales en 
contratos predispuestos y el mito de la baja calidad de los contratos de consumo, Derecho PUCP, 74, 249-267.

Slawson, W. (1971). Standard Form Contracts and Democratic Control of Law Making Power. Harvard Law Review, 84(3), 529-566. Recuperado de https://www.jstor. org/stable/1339552

Superintendencia de Banca, Seguros y Administradoras de Fondos de Pensiones. (2008) Resolución sBs N. 9756-2008.

_-_. (2008) Resolución sBs N. ${ }^{\circ}$ 10580-2008.

—_-. (2009) Resolución sBs N. 7835-2009.

_-_. (2009) Resolución sBs N.o 10519-2009.

———. (2009) Resolución sBs N. 9 9871-2009.

——_. (2009) Resolución sBs N. 9 9855-2009.

_-_. (2009) Resolución sBs N. ${ }^{\circ}$ 4552-2009.

_-_. (2009) Resolución sBs N. ${ }^{\circ}$ 4708-2009.

_-_. (2009) Resolución sBs N. 2185- 2009.

_-_. (2009) Resolución sBs N.o 10367-2009.

———. (2009) Resolución sBs N. ${ }^{\circ}$ 8948-2009.

_-_. (2009) Resolución sBs N. 3233-2009.

_-_. (2009) Resolución sBs N. 7017-2009.

——_. (2009) Resolución sBs N. 1101-2009.

_-_. (2009) Resolución sBs N. ${ }^{\circ}$ 1449-2009.

——_. (2009) Resolución sBs N. 7835-2009.

_-_. (2009) Resolución sBs N. . 4282-2009.

_-_. (2009) Resolución sBs N.o 10367-2009.

_-_. (2009) Resolución sBs N. 8948-2009.

_-_. (2009) Resolución sBs S N.o 13055-2009.

_-_. (2009) Resolución sBs N. ${ }^{0}$ 13006-2009.

—_—. (2009) Resolución sBs N. 13007-2009.

_-_. (2009) Resolución sBs N. o 9864- 2009.

_-_. (2009) Resolución sBs N. ${ }^{\circ}$ 130011-2009.

_-_. (2009) Resolución sBs N. ${ }^{0}$ 13008-2009.

—_—. (2009) Resolución sBs N.o 13613-2009.

_-_. (2009) Resolución sBs N.o 13614-2009.

——_. (2009) Resolución sBs N. 2416-2009.

_-_. (2009) Resolución sBs N. ${ }^{\circ}$ 2655-2009.

—_—. (2009) Resolución sBs N. ${ }^{o}$ 12340-2009.

———. (2009) Resolución sBs N. ${ }^{\circ}$ 9864-2009.

—_—. (2009) Resolución sBs N. ${ }^{0}$ 13006-2009.

_-_. (2009) Resolución sBs N. ${ }^{0}$ 12340-2009.

—_—. (2009) Resolución sBs N. 13616-2009.

_-_. (2009) Resolución sBs N.o 13615-2009.

_——. (2009) Resolución sBs N. ${ }^{\circ}$ 13007-2009.
———. (2009) Resolución sBs N. o 130088-2009.

——_. (2009) Resolución SBs N. ${ }^{\circ}$ 131388-2009.

_-_. (2009) Resolución sBs N. o 13514-2009.

———. (2009) Resolución sBs N. ${ }^{\circ}$ 14212-2009.

_-_. (2009) Resolución sBs N.o 15211-2009.

———. (2009) Resolución SBs S N. ${ }^{\circ}$ 15564-2009.

———. (2009) Resolución sBs N. o 14118-2009.

_—_. (2011) Resolución SBs N. ${ }^{\circ}$ 1425-2011.

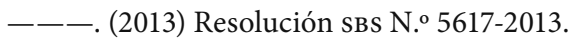

_-_. (2013) Resolución sBs N. ${ }^{0}$ 5254-2013.

———. (2013) Resolución sBs N. ${ }^{\circ}$ 6860-2013.

——_. (2014) Resolución sBs N. ${ }^{\circ} 4050-2014$.

_-—. (2014) Resolución sBs N. 3 3481-2014.

———. (2014) Resolución sBs N. ${ }^{\circ}$ 3535-2014.

———. (2014) Resolución SBs N. ${ }^{\circ}$ 8149-2014.

———. (2014) Resolución sBs S N. 7778-2014.

———. (2014) Resolución sBs N. o 3819-2014.

———. (2014) Resolución sBs N. ${ }^{\circ}$ 8253-2014.

_-_. (2014) Resolución sBs N. . 5792-2014.

———. (2014) Resolución sBs N. ${ }^{\circ}$ 5791-2014.

- - - (2014) Resolución sBs N. 5228-2014.

_-_. (2014) Resolución sBs No 6250-2014.

- - - (2015) Resolución sBs N. 9 942-2015.

- - - (2015) Resolución sBs N. ${ }^{\circ}$ 2622-2015.

———. (2015) Resolución sBs N. ${ }^{o}$ 1942-2015.

———. (2015) Resolución sBs N. ${ }^{\circ}$ 7333-2015.

———. (2015) Resolución sBs N. ${ }^{0}$ 7126-2015.

———. (2015) Resolución sBs N. ${ }^{\circ}$ 4806-2015.

———. (2015) Resolución sBs N. ${ }^{o}$ 7069-2015.

———. (2015) Resolución sBs N. ${ }^{\circ}$ 1066-2015.

———. (2015) Resolución sBs S N. o 2622-2015.

———. (2016) Resolución sBs N. ${ }^{\circ}$ 5575-2016.

_-_. (2016) Resolución sBs N. ${ }^{o}$ 5942-2016.

———. (2016) Resolución sBs N. 3522-2016.

_-_. (2016) Resolución sBs N. ${ }^{\circ}$ 4435-2016.

———. (2017) Resolución SBs N. ${ }^{01820-2017 . ~}$

———. (2018) Resolución sBs N.o 1684-2018.

———. (2018) Resolución sBs N. ${ }^{\circ}$ 357-2018.

———. (2018) Resolución sBs N. ${ }^{\circ} 2122-2018$.

- - - (2018) Resolución sBs N. ${ }^{\circ}$ 2000-2018.

- - - (2019) Resolución sBs N.o 2202-2019.

- - - (2019) Resolución sBs N. 2189-2019.

- - - (2019) Resolución sBs N.o 2201-2019.

Criterios orientadores de la incorporación de condiciones generales en los contratos financieros

y la tutela del consumidor peruano 
———. (2019) Resolución sBs N. 9 933-2019.

———. (2019) Resolución sBs N.o 2184-2019

-——. (2019) Resolución sBs N. * 3626-2019

Thaler, R. S. (2003). Libertarian Paternalism is not an Oxymoron (University of Chicago Public Law \& Le- gal Theory Working Paper n. $\left.{ }^{\circ} 43\right)$. Recuperado de https://chicagounbound.uchicago.edu/cgi/viewcontent $/$.cgi? article $=1184 \&$ context $=$ pubic_law_and_legal_theory

Torres Vásquez, A. (2016). Teoría general del contrato (vol. I, 2a ed.). Lima: 2016. 